\title{
Correction: Transmission of Specific Genotype Streptomycin Resistant Strains of Mycobacterium tuberculosis in the Tokyo Metropolitan Area in Japan
}

Akihiro Ohkado ${ }^{1 *}$, Yoshiro Murase ${ }^{2}$, Masaaki Mori ${ }^{3}$, Naoki Hasegawa ${ }^{4}$, Goro Otsuka ${ }^{5}$, Michiko Nagamine ${ }^{6}$, Hideo Maeda ${ }^{6}$, Kazuhiro Uchimura ${ }^{1}$, Masako Ohmori ${ }^{1}$, Norio Yamada ${ }^{7}$, Shinji Maeda ${ }^{2}$, Seiya Kato ${ }^{8}$, Toru Mori ${ }^{8}$, Nobukatsu Ishikawa ${ }^{8}$

\section{Correction}

We noticed a one-digit error on the tandem repeat number at the MIRU 20 after the publication of our article [1]. This was due to a typing error. The tandem repeat number at the MIRU 20 should be 2 instead of 1 . All the figures indicating the tandem repeat number at the MIRU 20, i.e. the fifth digit of the VNTR profile from the left end of the set of digits, should be corrected in the text and correct information is provided in the revised Table 1 . The first 12 digits of the VNTR profiles in the Methods part and Results part as well as in the Table 1 should read as 223325173533instead of 223315173533- (Table 1).
We do apologize to readers for any misunderstanding or inconvenience caused by this error.

\section{Author details}

'Department of Epidemiology and Clinical Research, Research Institute of Tuberculosis, Japan Anti-Tuberculosis Association, Matsuyama 3-1-24, Kiyose, Tokyo, Japan. ${ }^{2}$ Department of Reference for Mycobacteria, Research Institute of Tuberculosis, Japan Anti-Tuberculosis Association, Kiyose, Tokyo, Japan. ${ }^{3}$ Health Centre, Keio University, Yokohama, Japan. ${ }^{4}$ Department of Respiratory Medicine, Keio University, Yokohama, Japan. ${ }^{5}$ Department of Health and Clinical Medicine, Bureau of Health and Welfare, Kawasaki, Japan. ${ }^{6}$ Bureau of Social Welfare and Public Health, Tokyo Metropolitan Government, Shinjuku, Tokyo, Japan.

${ }^{7}$ Department of International Cooperation, Research Institute of Tuberculosis, Japan Anti-Tuberculosis Association, Kiyose, Tokyo, Japan. ${ }^{8}$ Research Institute of Tuberculosis, Japan Anti-Tuberculosis Association, Kiyose, Tokyo, Japan.

\footnotetext{
* Correspondence: ohkadoa@jata.or.jp

'Department of Epidemiology and Clinical Research, Research Institute of

Tuberculosis, Japan Anti-Tuberculosis Association, Matsuyama 3-1-24, Kiyose, Tokyo, Japan
}

(c) 2010 Ohkado et al; licensee BioMed Central Ltd. This is an Open Access article distributed under the terms of the Creative Commons 
Table 1 Patient and VNTR Profiles whose M.tuberculosis Indicated M-strains

\begin{tabular}{|c|c|c|c|c|c|c|c|c|c|}
\hline No. & $\begin{array}{c}\text { M-strain } \\
\text { Type } \\
(* 1)\end{array}$ & $\begin{array}{c}\text { Year } \\
\text { Patient } \\
\text { Registered }\end{array}$ & $\begin{array}{c}\text { TB } \\
\text { Diagnosis } \\
(* 2)\end{array}$ & $\begin{array}{l}\text { Sex } \\
(* 3)\end{array}$ & $\begin{array}{c}\text { Age } \\
\text { (years old) }\end{array}$ & $\begin{array}{c}\text { Internet-café } \\
\text { Use } \\
(* 4)\end{array}$ & $\begin{array}{c}\text { Homeless } \\
\left({ }^{*} 5\right)\end{array}$ & $\begin{array}{c}\text { Drug } \\
\text { Resistance } \\
\left({ }^{* 6)}\right.\end{array}$ & $\begin{array}{c}\text { VNTR profiles } \\
\left({ }^{* 7)}\right.\end{array}$ \\
\hline 1 & M5 & 2004 & univ & M & 22 & No & No & SM & 223325173533-4253-8844434-4337 \\
\hline 2 & M5 & 2004 & univ & M & 22 & No & No & SM & 223325173533-4253-8844433-4337 \\
\hline 3 & M5 & 2004 & univ & M & 22 & No & No & SM & $223325173533-4253-8844434-4337$ \\
\hline 4 & M5 & 2004 & univ & M & 22 & Yes & No & SM & 223325173533-4253-8844434-4337 \\
\hline 5 & M5 & 2004 & univ & $\mathrm{F}$ & 21 & No & No & SM & $223325173533-4253-8844433-4337$ \\
\hline 6 & M5 & 2004 & univ & M & 22 & No & No & SM & 223325173533-4253-8844434-4337 \\
\hline 7 & M5 & 2004 & univ & M & 22 & No & No & SM & $223325173533-4253-8844433-4337$ \\
\hline 8 & M5 & 2004 & univ & M & 22 & No & No & SM & $223325173533-4253-8844433-4337$ \\
\hline 9 & M5 & 2004 & univ & M & 22 & No & No & SM \& INH & $223325173533-4253-8844434-4337$ \\
\hline 10 & M5 & 2004 & univ & M & 22 & No & No & $\mathrm{SM} \& \mathrm{INH}$ & 223325173533-4253-8844434-4337 \\
\hline 11 & M5 & 2004 & univ & M & 22 & No & No & SM & 223325173533-4253-88244334-4337 \\
\hline 12 & M5 & 2005 & univ & $\mathrm{F}$ & unknown & No & No & SM & $223325173533-4253-8844434-4337$ \\
\hline 13 & M5 & 2005 & univ & M & 22 & No & No & SM & $223325173533-4253-8844434-4337$ \\
\hline 14 & M5 & 2005 & univ & M & 24 & No & No & SM & $223325173533-4253-8844433-4337$ \\
\hline 15 & M5 & 2005 & univ & M & 21 & No & No & SM & 223325173533-4253-8844434-4337 \\
\hline 16 & M5 & 2005 & univ & M & 22 & No & No & SM & $223325173533-4253-8844434-4337$ \\
\hline 17 & M5 & 2005 & univ & M & 23 & No & No & SM & $223325173533-4253-8844433-4337$ \\
\hline 18 & M5 & 2005 & univ & M & 22 & No & No & SM & 223325173533-4253-8844434-4337 \\
\hline 19 & M5 & 2006 & s & M & 58 & No & Yes & SM & 223325173533-4253-8844433-4337 \\
\hline 20 & M5 & 2007 & $\mathrm{~s}$ & M & 39 & Yes & Yes & SM & $223325173533-4253-8844433-4337$ \\
\hline 21 & M4 & 2003 & $\mathrm{~s}$ & M & 59 & No & No & SM & $223325173533-42 \underline{2} 3-884443 \underline{3}-4337$ \\
\hline 22 & M4 & 2004 & univ & M & 22 & Yes & No & SM & $223325173533-4243-884443 \underline{3}-4337$ \\
\hline 23 & M4 & 2004 & univ & M & 24 & No & No & SM & $223325173533-4243-884443 \underline{3}-4337$ \\
\hline 24 & M4 & 2004 & $\mathrm{Ob}$ & M & 24 & No & No & SM & $223325173533-42 \underline{-} 3-884443 \underline{3}-4337$ \\
\hline 25 & M4 & 2004 & $\mathrm{Ob}$ & M & 35 & No & No & SM & $223325173533-42 \underline{2} 3-884443 \underline{3}-4337$ \\
\hline 26 & M4 & 2004 & ka & M & 49 & No & Yes & SM & $223325173533-4243-884443 \underline{-}-4337$ \\
\hline 27 & M4 & 2004 & ka & M & 31 & No & No & SM & $223325173533-4243-884443 \underline{3}-4337$ \\
\hline 28 & M4 & 2004 & ka & M & 41 & No & No & SM & $223325173533-4243-8844433-4337$ \\
\hline 29 & M4 & 2004 & s & M & 65 & Yes & Yes & SM & $223325173533-4243-884443 \underline{3}-4337$ \\
\hline 30 & M4 & 2005 & $\mathrm{Ob}(\mathrm{ka})$ & M & 47 & Yes & Yes & SM & 223325173533-4243-8844433-4337 \\
\hline 31 & M4 & 2005 & $\mathrm{Ob}(\mathrm{s})$ & M & 28 & Yes & No & SM & $223325173533-4243-8844433-4337$ \\
\hline 32 & M4 & 2005 & $\mathrm{Ob}$ & M & 31 & Yes & Yes & SM & $223325173533-42 \underline{2} 3-884443 \underline{3}-4337$ \\
\hline 33 & M4 & 2005 & $\mathrm{Ob}$ & M & 25 & Yes & Yes & SM & $223325173533-42 \underline{2} 3-884443 \underline{3}-4337$ \\
\hline 34 & M4 & 2005 & ka & M & 30 & No & No & SM & $223325173533-4243-884443 \underline{3}-4337$ \\
\hline 35 & M4 & 2005 & ka & $\mathrm{F}$ & 56 & No & No & SM & $223325173533-4243-8844433-4337$ \\
\hline 36 & M4 & 2005 & ka & M & 45 & Yes & Yes & SM & 223325173533-4243-8844433-4337 \\
\hline 37 & M4 & 2006 & ka & M & 67 & No & No & SM & $223325173533-4243-8844433-4337$ \\
\hline 38 & M4 & 2006 & ka & M & 58 & No & No & SM & $223325173533-4243-8844433-4337$ \\
\hline 39 & M4 & 2006 & s & $\mathrm{F}$ & 33 & No & No & SM & $223325173533-4243-8844433-4337$ \\
\hline 40 & M4 & 2007 & ka & M & 46 & No & No & SM & $223325173533-42 \underline{2} 3-884443 \underline{3}-4337$ \\
\hline 41 & M4 & 2007 & ka & M & 54 & No & No & SM & $223325173533-4243-8844433-4337$ \\
\hline 42 & M4 & 2007 & ka & M & 44 & No & No & SM & $223325173533-4243-\underline{6} 84443 \underline{3}-4337$ \\
\hline 43 & M4 & 2007 & ka & $\mathrm{F}$ & 39 & No & No & SM & $223325173533-4243-8844433-4337$ \\
\hline
\end{tabular}


Table 1: Patient and VNTR Profiles whose M.tuberculosis Indicated M-strains (Continued)

\begin{tabular}{|c|c|c|c|c|c|c|c|c|c|}
\hline 44 & M4 & 2007 & ka & M & 55 & No & No & SM & $223325173533-4243-884443 \underline{3}-4337$ \\
\hline 45 & M4 & 2007 & s & M & 50 & Yes & Yes & SM & $223325173533-4243-884443 \underline{3}-4337$ \\
\hline 46 & M4 & 2007 & $\mathrm{~s}$ & M & 45 & No & Yes & SM & $223325173533-4243-8844433-4337$ \\
\hline
\end{tabular}

*1: "M4" indicates the M4-substrain with four tandem repeat copies at the ETR C locus. "M5" indicates the M5-substrain with five tandem repeat copies at the ETR C locus.

*2: "univ" indicates a TB patient detected through the contact investigation related to the tuberculosis outbreak at the university campus mentioned in the text. "Ob" indicates a TB patient detected through contact investigations related to tuberculosis outbreak incidents other than the one at the university campus mentioned above.

"s" indicates a TB patient detected through the population-based DNA fingerprinting surveillance of M.tuberculosis in Shinjuku City.

"ka" indicates a TB patient detected through the hospital-based DNA fingerprinting surveillance of M.tuberculosis in Kawasaki City.

*3: " $M$ " indicates male and " $F$ " indicates female.

*4: "Yes" indicates a TB patient who claimed to have a past history of the use of some internet cafés in the TMA within the past two years.

*5: A homeless person is defined as a person whose legal address is unknown or unstable at least within the past two years.

*6: Anti-tuberculosis agents the isolated M.tuberculosis indicated resistance. "SM" stands for Streptomycin, "INH" stands for Isoniazid.

*7: The 12 digits at the first part of the VNTR profile indicate the tandem repeat copy numbers according to the following loci set order: MIRU2, MIRU4, MIRU10, MIRU16, MIRU20, MIRU23, MIRU24, MIRU26, MIRU27, MIRU31, MIRU39, MIRU40 (Reference 5).

The 4 digits at the second part of the VNTR profile indicate the tandem repeat copy numbers according to the following loci set order: ETR A, ETR B, ETR C, ETR $\mathrm{F}$ (Reference 6).

The 7 digits at the third part of the VNTR profile indicate the tandem repeat copy numbers according to the following loci set order: QUB11b, QUB26, Mtub04, Mtub21, Mtub30, Mtub39, VNTR4156 (Reference 7).

The 4 digits at the last part of the VNTR profile indicate the tandem repeat copy numbers according to the following loci set order: QUB15, Mtub24, VNTR2372, VNTR3336 (Reference 8).

Received: 1 March 2010 Accepted: 29 March 2010

Published: 29 March 2010

\section{Reference}

1. Ohkado Akihiro, Murase Yoshiro, Mori Masaaki, Hasegawa Naoki,

Otsuka Goro, Nagamine Michiko, Maeda Hideo, Uchimura Kazuhiro,

Ohmori Masako, Yamada Norio, Maeda Shinji, Kato Seiya, Mori Toru,

Ishikawa Nobukatsu: Transmission of Specific Genotype Streptomycin

Resistant Strains of Mycobacterium tuberculosis in the Tokyo

Metropolitan Area in Japan. BMC Infectious Diseases 2009, 9:138.

\section{Pre-publication history}

The pre-publication history for this paper can be accessed here: http://www. biomedcentral.com/1471-2334/10/79/prepub

\section{doi:10.1186/1471-2334-10-79}

Cite this article as: Ohkado et al:: Correction: Transmission of Specific Genotype Streptomycin Resistant Strains of Mycobacterium tuberculosis in the Tokyo Metropolitan Area in Japan. BMC Infectious Diseases 2010

$10: 79$.

\section{Submit your next manuscript to BioMed Central and take full advantage of:}

- Convenient online submission

- Thorough peer review

- No space constraints or color figure charges

- Immediate publication on acceptance

- Inclusion in PubMed, CAS, Scopus and Google Scholar

- Research which is freely available for redistribution

\section{西}

